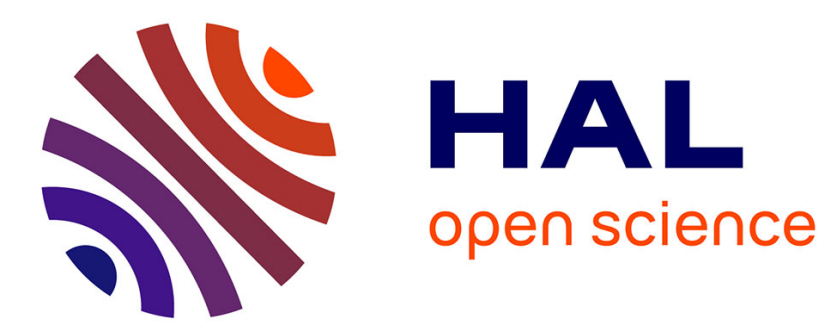

\title{
On helicoidal ends of minimal surfaces
}

Pascal Romon

\section{- To cite this version:}

Pascal Romon. On helicoidal ends of minimal surfaces. Annals of Global Analysis and Geometry, 1994, 12 (1), pp.341. 10.1007/BF02108306 . hal-00860937

\section{HAL Id: hal-00860937 https://hal.science/hal-00860937}

Submitted on 11 Sep 2013

HAL is a multi-disciplinary open access archive for the deposit and dissemination of scientific research documents, whether they are published or not. The documents may come from teaching and research institutions in France or abroad, or from public or private research centers.
L'archive ouverte pluridisciplinaire $\mathbf{H A L}$, est destinée au dépôt et à la diffusion de documents scientifiques de niveau recherche, publiés ou non, émanant des établissements d'enseignement et de recherche français ou étrangers, des laboratoires publics ou privés. 


\title{
ON HELICOIDAL ENDS OF MINIMAL SURFACES
}

\author{
PASCAL ROMON \\ Centre de Mathématiques et Leurs Applications \\ Unité de Recherche Associée CNRS 1611 \\ École Normale Supérieure de Cachan \\ 61 avenue du Président Wilson \\ 94235 Cachan Cedex, FRANCE
}

\begin{abstract}
This article analyses the behaviour of helicoidal ends of properly embedded minimal surfaces, namely properly embedded infinite total curvature minimal annuli of parabolic type, satisfying a growth condition on the curvature via the Gauss map, and a geometric transversality condition. Then we show that embeddedness forces the end to be asymptotic either to a plane, or a helicoid or a spiraling helicoid. In all three cases, the Gauss map can be described in very simple terms. Finally this local result yields a global corollary stating the rigidity of embedded minimal helicoids.
\end{abstract}

Key words: minimal surface, infinite total curvature, annular end, helicoid, embeddedness, essential singularity.

MSC classification: $53 \mathrm{~A} 10$.

\section{Introduction}

In this paper we tackle a relatively new problem in minimal surface theory (or rather an old problem with a new approach): the problem of embeddedness for surfaces with infinite total curvature. Indeed, most of the minimal surfaces discovered so far have been of finite total curvature (see [10] for a good overview), and this condition has been crucial in their finding and understanding. However surfaces with infinite total curvature, such as the helicoid, have been known to exist since the very beginning of the theory. Obviously, all periodic minimal examples but the plane have infinite total curvature (and often infinite genus too), although they were often studied in $\mathbb{R}^{3}$ modulo periods, thus becoming finite total curvature and finite 
genus examples $([6])$. It was not known until recently whether there could exist a non-periodic surface, which was truly of infinite total curvature. Eventually D. Hoffman, H. Karcher and F. Wei proved the existence of genus one "helicoid" (see [4]) ${ }^{1}$. Conversely some non-existence results have been obtained for the two-ended case and the Nitsche conjecture ([1], [10]). It it thus quite natural to wonder which such surfaces can be embedded in $\mathbb{R}^{3}$ and this is what this article is concerned about.

The idea behind the results presented hereafter is to generalize the theorem of Osserman [7] linking finite total curvature to finite topology:

Theorem [Osserman] : Let $M$ be a complete minimal surface in $\mathbb{R}^{3}$ with finite total curvature; then $M$ is conformally equivalent to a closed Riemann surface $\tilde{M}$ with a finite number of punctures. Furthermore the Enneper-Weierstrass data extends meromorphically on $\tilde{M}$.

We hope indeed to show that embedded minimal surfaces of finite topological type with infinite total curvature - provided the curvature does not grow too fast - are described by simple algebraic data $\frac{d g}{g}$ where $g$ is the corresponding Gauss map. Indeed such is the case for helicoidal ends as we will see in the following. Notice that the hypothesis of embeddedness is essential, as will become clear later on (see also [12]). More information on this topic can be found in [11].

We take the example of the helicoid as a starting point. It can be parameterized globally by the Weierstrass-Enneper data $(g, \eta)$ through $X(z)=\operatorname{Re} \int^{z}\left(g^{-1}-g, i\left(g^{-1}+g\right), 2\right) \eta$, where $g$ and $\eta$ are respectively a meromorphic function and a méromorphic 1-form on the complex plane. Then

$$
\left\{\begin{array}{l}
g=e^{z} \\
\eta=i d z
\end{array}\right.
$$

describes the helicoid. There are two ways of generalizing the family of the helicoid: one is to find minimal embeddings of the complex plane, the other is to understand locally the behavior of the end, when the end is conformally a punctured annulus. In both cases we will impose the following geometric condition: the end $E$ intersects each horizontal plane in a single open curve. That implies that, up to a reparameterization, the 1-form $\eta$ is just $i d z$ (as in the case of the helicoid); consequently, the tangent plane is never horizontal, and the Gauss map $g$ never takes the values 0 or $\infty$.

Because the analysis of meromorphic data on $\mathbb{C}$ is largely determined by the behavior at infinity, the second approach encompasses the first one, and gives additional information too. We will now state the results:

Theorem 1 (local) Let $M$ be a properly embedded minimal surface in $\mathbb{R}^{3}$, conformally equivalent to a punctured disk, such that its intersection with any horizontal plane is transversal and consists of either one curve diffeomorphic to $\mathbb{R}$, or two half-curves diffeomorphic to $\mathbb{R}_{+}$(up to a rotation of the surface). If the Gauss map $g$, seen as a meromorphic map through the

\footnotetext{
${ }^{1}$ Its end is asymptotic to a helicoid, hence embedded. It not proven yet whether the whole surface is embedded.
} 
stereographic projection, has finite order in the sense of Nevanlinna, then $X$ is asymptotic to a spiraling helicoid, or the helicoid, or the plane.

Corollary 2 (global) Let $M$ be a properly embedded minimal plane in $\mathbb{R}^{3}$, which meets all but a finite number of horizontal planes (up to a rotation in space) transversely in a single open curve. If the Gauss map g, seen as a meromorphic map through the stereographic projection, has finite order in the sense of Nevanlinna, then $X$ is the helicoid or the plane.

\section{The behavior of helicoidal ends}

Let $X$ be an immersion from the punctured disk centered at infinity $D_{r_{0}}^{\infty}=\left\{z \in \mathbb{C} ;|z| \geq r_{0}\right\}$, satisfying the hypotheses of theorem 1 . We contend that up to reparameterization, we have $\eta=i d z$. To prove that just apply the following lemma to $X_{3}$ :

Lemma 1 Let $u$ be a proper harmonic map defined on $D_{r_{0}}^{\infty}$ such that the preimage $u^{-1}(c)$ is diffeomorphic either to $\mathbb{R}$ or to two copies of $\mathbb{R}_{+}$. Then, up to reparametrization, $u$ is linear.

PROOF : Take any strip of the surface $X_{3}^{-1}([c, c+1])$; for $c$ large enough, it is bounded by two curves. Its conformal type is a disk minus two disjoint curves $\alpha$ and $\beta$; we claim that these curves are actually just points. Heuristically, we see that going to infinity on the strip is just a way of going to the puncture in $D_{r_{0}}^{\infty}$. To prove this assertion consider a half-strip $\Sigma$ in $D_{r_{0}}^{\infty}$, and define a harmonic map on $\Sigma$ going to infinity at the end, namely $z \mapsto \log |z|$. By simple connectivity there exists a holomorphic map $f$ having $\log |z|$ as real part; if the boundary at infinity of $\Sigma$ were conformally a curve $\alpha$ (seen in the Poincaré disk for instance) then $f$ would be infinite along a curve, which is impossible. So the conformal type of any strip $X_{3}^{-1}([a, b])$ for $|a|,|b|$ large enough, is a disk minus two points; furthermore these two points correspond to the same point at infinity in $D_{r_{0}}^{\infty}$. In particular we map the strip conformally to $\Omega=\{z \in \mathbb{C} ; 0 \leq \operatorname{Im}[z] \leq 1\}$.

We conclude by using the Phragmén-Lindelöf theorem (see [13]) : assume for simplicity that $X_{3}(z)=0$ (respectively 1 ) if $\operatorname{Im}[z]=0$ (respectively 1 ); let $\phi$ be a holomorphic mapping from the strip such that $X_{3}=\operatorname{Re}[\phi]$, and $F=e^{\phi+i z}$. Obviously $|F|=e^{X_{3}(z)-\operatorname{Im}[z]}$ is bounded on $\Omega$, and equal to one on $\partial \Omega$. Then we can use an extended maximum principle, and state that $|F| \equiv 1$ on $\Omega$, proving that $X_{3}$ coincides with $-\operatorname{Im}[z]$, and $\eta$ with $i d z$.

As a consequence, we claim that the Gauss map never takes the values 0 and $\infty$. Indeed the metric $d s^{2}=\left(|g|+|g|^{-1}\right)^{2}|\eta|^{2}$ has to be well defined and non-zero. As will be seen later on, $g$ can be written as $z^{k} e^{f}$ for some integer $k$ and some holomorphic function $f$ on $D_{r_{0}}^{\infty}$.

Let us study the geometry of the level curves, which correspond in our parameterization to the map $t \mapsto i l+t$, for fixed $l$. The normal vector to the curve is obviously $\nu=g /|g|$, identifying the plane of the curve with the complex plane. Then the Frenet frame is $(\tau, \nu)=\left(-i \frac{g}{|g|}, \frac{g}{|g|}\right)$. 
We can now derive the curvature; notice that the derivative $\frac{d}{d t}$ along the curve coincides with the complex derivative.

$$
\left\langle\frac{d \tau}{d t}, \nu\right\rangle=\operatorname{Im}\left[\frac{g^{\prime}}{g}\right]
$$

where $\langle$,$\rangle is the standard metric in \mathbb{R}^{2} \simeq \mathbb{C}$. Denoting by $s$ the arclength,

$$
\kappa(t)=\left\langle\frac{d \tau}{d s}, \nu\right\rangle=\frac{d t}{d s}\left\langle\frac{d \tau}{d t}, \nu\right\rangle=\frac{\left\langle\frac{d \tau}{d t}, \nu\right\rangle}{\left(|g|^{-1}+|g|\right)}
$$

So

$$
\kappa(t)=\frac{\operatorname{Im}\left[\frac{g^{\prime}}{g}\right]}{|g|^{-1}+|g|}
$$

From now on we assume that the function $f$ defined above has bounded degree at infinity, i.e. $f \in O\left(z^{\alpha}\right)$ for some $\alpha>0$ (in other words $f$ is sum of a polynomial and a holomorphic term vanishing at infinity). We let $n$ denote the degree of $f$, and suppose that $n \geq 2$. Since we are interested in horizontal curves $\operatorname{Im} z=l$ for constant $l$, we will restrict ourselves to non-negative $l$; we can then define on the upper half plane a complex logarithm and write $g=e^{H}$ with $H(z)=f(z)+k \log z$. Also $h$ will be the derivative $H^{\prime}$. We rewrite (1) as

$$
\kappa(t)=\frac{2 \operatorname{Im}[h]}{\cosh \operatorname{Re}[H]}
$$

then the curve $\xi_{l}(t)=\left(X_{1}+i X_{2}\right)(i l+t)$ satisfies the equation

$$
\frac{d \xi_{l}}{d t}=\frac{-i}{2} \cosh \operatorname{Re}[H] e^{i \operatorname{Im}[H]}
$$

The curves $\xi_{l}$ may be very complex, and increasingly complex as $n$ grows. However we are able to show a very simple asymptotic behavior. For this we will need the

Lemma 2 For any $\alpha$ define $x_{\alpha}(t)=\left\langle\xi_{l}(t), e^{i \alpha}\right\rangle$. Let $t_{n}$ be the sequence of local maxima (resp. minima) of $x_{\alpha}$. For $|l|$ large enough, there exists $\hat{t}$ independent of $\alpha$ such that the sequence $x_{\alpha}\left(t_{n}\right)$ is increasing (resp. decreasing) to infinity, when $t>\hat{t}$.

PROOF : Let $A(t), B(t)$ denote the real and imaginary parts of $H(i l+t)$ respectively; then the extrema of $x_{\alpha}$ are exactly the zeros of $\sin (B(t)-\alpha)$. We will suppose always that $\alpha$ is chosen in $\left[0,2 \pi\left[\right.\right.$. Moreover $A(t) \sim A_{0} t^{a}$ and $B(t) \sim B_{0} t^{b}$ for some non-negative ${ }^{2}$ integers $a$ and $b$, since $\operatorname{deg}(f)=n \geq 2$. Since $b>0$, obviously $B$ is strictly increasing or decreasing for some $l$ large and $t$ large, independently of $\alpha$. Say for simplicity that $B$ is increasing, then $B$ is a diffeomorphism for large $t$; let $\psi$ be its inverse. Finally let

$$
u_{k}=\psi(k \pi+\alpha)
$$

\footnotetext{
${ }^{2}$ However it is possible for $B$ to "drop in degree", i.e. let $b=0$ for a finite set of values of $l$.
} 
correspond to the $k$-th extremum for large $k \in \mathbb{N}$. Then the variation of $x_{\alpha}$ between two successive extrema is:

$$
\Delta_{k}=\frac{1}{2} \int_{u_{k}}^{u_{k+1}} \cosh A(u) \sin (B(u)-\alpha) d u=\frac{1}{2} \int_{k \pi}^{(k+1) \pi} \cosh (\psi(t+\alpha)) \sin (t) \psi^{\prime}(t+\alpha) d t
$$

We want to compare it with $\Delta_{k-1}$ using a change of variable

$$
\Delta_{k}=-\frac{1}{2} \int_{(k-1) \pi}^{k \pi} \cosh (\psi(t+\alpha+\pi)) \sin (t) \psi^{\prime}(t+\alpha+\pi) d t
$$

Since $a>0, \cosh A(\psi(u)) \psi^{\prime}(u)$ is a positive increasing function and so is its derivative; then for all $u$ greater than some $\hat{u}$.

$$
\cosh A(\psi(u+\pi)) \psi^{\prime}(u+\pi) \geq 2+\cosh A(\psi(u)) \psi^{\prime}(u)
$$

by the mean value theorem. So that $\Delta_{k}$ and $\Delta_{k-1}$ always have opposite signs and $\left|\Delta_{k}\right| \geq$ $1+\left|\Delta_{k-1}\right|$ for all $k \geq \hat{k}$. We conclude that the maximal values (resp. minimal) of $x_{\alpha}$ increase (resp. decrease) towards infinity.

Using this lemma, we can prove that $\xi_{l}$ is asymptotically a diverging spiral, i.e. there exist functions $\rho$ and $\theta$ both going to infinity, $\theta$ strictly monotone, such that $\xi_{l}(t)=\rho(t) e^{i \theta(t)}$, when $t$ is large enough. To construct those functions consider a ray from the origin, with angle $\theta$. Using the intermediate values theorem, we deduce from the previous lemma that the ray meets our curve a infinite number of time, at least for $t>\hat{t}$ (just consider $\alpha=\theta+\pi / 2$ ). If the ray does not meet the curve tangentially, we can parameterize locally in polar coordinates. If that is not so, then $x_{\theta+\pi / 2}$ has an extremum at that point, with a value of zero. This contradicts again the lemma, so we can parametrize globally in polar coordinate, which proves that $\theta$ is strictly monotonic. Suppose now that we can find $t_{1}$ arbitrarily large, such that $\rho\left(t_{1}\right)$ is smaller than some fixed value $\tilde{\rho}$. Let $e^{i \theta_{1}}$ be a tangent vector to $\xi_{l}$ at $t_{1}$, then $x_{\theta_{1}+\pi / 2}$ has an extremum at $t_{1}$; but we know that for $t$ big enough, $\left|x_{\theta_{1}+\pi / 2}(t)\right|>\tilde{\rho}$.

We now have a good description of the asymptotic behavior of the curve $\xi_{l}$ for almost all $l$. Both infinite branches are spirals.

REMARK 1 : If $b=\operatorname{deg}(B)$ is odd, the curve is obviously not embedded (see figure 1). Indeed both branches spiral to infinity with opposite winding directions; they are bound to meet each other infinitely many times. Indeed compare the two branches: $t \mapsto \xi_{l}(t)$ and $t \mapsto \xi_{l}(-t)$ when $t \rightarrow+\infty$. By the previous calculations we obtain almost identical results, except for the angle $\theta(t)$ : in one case it is increasing, in the other, decreasing (or just notice that the curvature $\kappa$ has the same sign when $t \rightarrow \pm \infty$ ). 


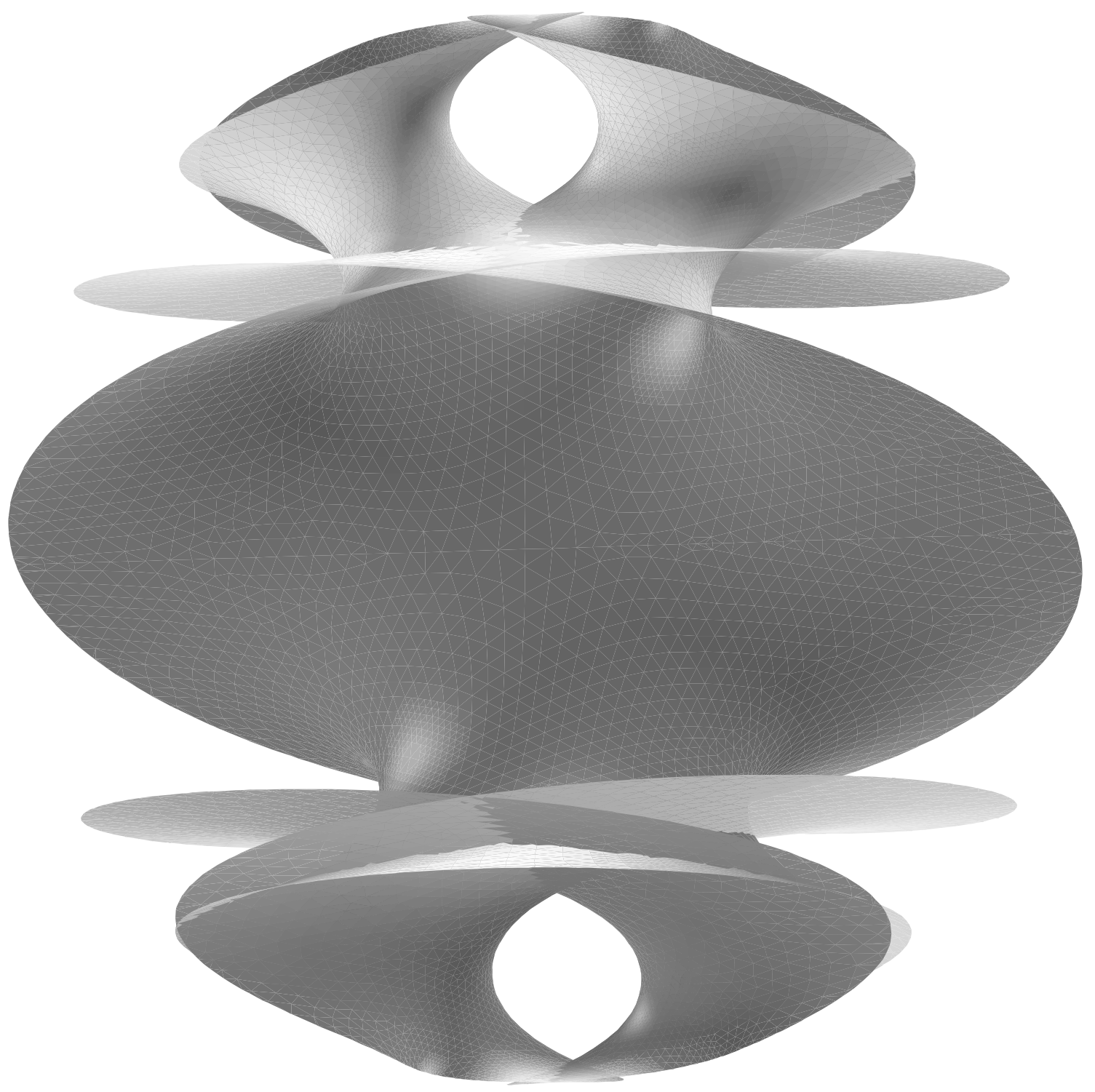

Figure 1: Example of non-embedded disk when $\operatorname{deg}(B)$ is odd. Here $g=e^{z^{2}}$ and $B(t)=2 i l t$. 


\section{A geometrical criterion for self-intersection}

We will now give a criterion for any plane curve that will ensure self-intersection. Let $\gamma$ be such a curve, parameterized by its tangential angle $\theta$ in complex coordinates; namely

$$
\frac{d \gamma}{d \theta}=\frac{e^{i \theta}}{\kappa(\theta)}
$$

where $\kappa$ is the curvature (of the curve).

Lemma 3 Let $\theta_{0}$ be such that

(i) $\kappa$ is non-negative increasing on $\left[\theta_{0}-\frac{3 \pi}{2}, \theta_{0}\right]$,

(ii) $\kappa$ is non-negative decreasing on $\left[\theta_{0}, \theta_{0}+\frac{3 \pi}{2}\right]$,

(iii) $\kappa(\pi) \leq \kappa\left(-\frac{\pi}{2}\right)$ and $\kappa(-\pi) \leq \kappa\left(\frac{\pi}{2}\right)$,

then $\gamma$ has a self-intersection between $\theta_{0}-\frac{3 \pi}{2}$ and $\theta_{0}+\frac{3 \pi}{2}$. The same is true if $\kappa$ is replaced by $-\kappa$.

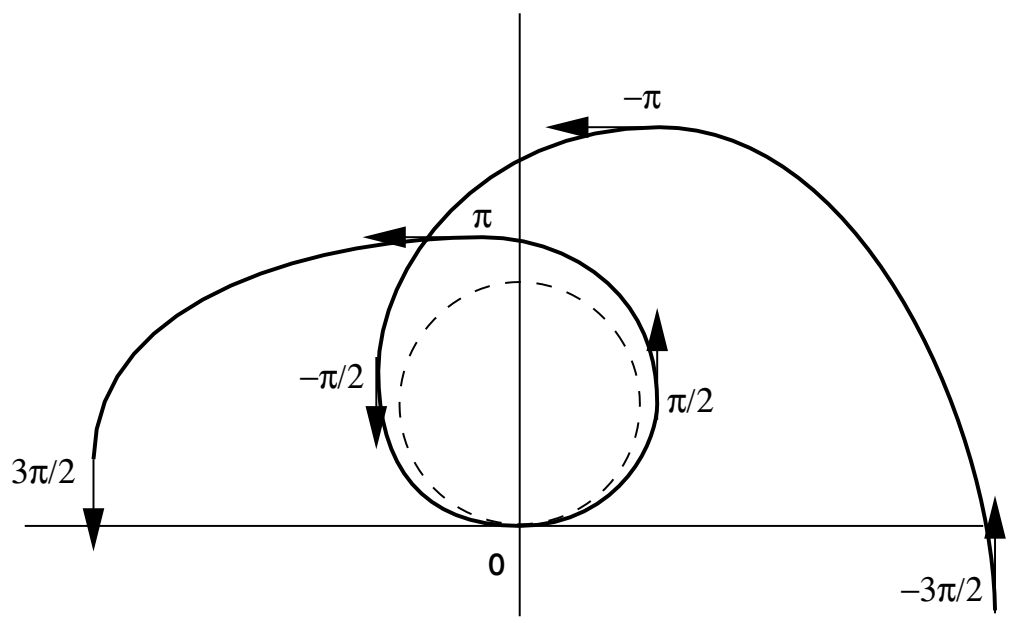

Figure 2: Typical case

PROOF : By rotating and translating the curve in the plane, we can assume that $\theta_{0}=0$ and $\gamma(0)=0$. Then hypothesis (i) (respectively (ii)) implies that $\operatorname{Re}[\gamma(-\pi)]$ is positive (respectively $\operatorname{Re}[\gamma(\pi)] \leq 0$ ), and both imaginary parts are positive. Indeed:

$$
\int_{0}^{\pi / 2} \frac{\cos \theta}{\kappa(\theta)} d \theta \leq \frac{1}{\kappa(\pi / 2)}
$$

while

$$
\int_{\pi / 2}^{\pi} \frac{|\cos \theta|}{\kappa(\theta)} d \theta \geq \frac{1}{\kappa(\pi / 2)}
$$


The same goes for $\gamma(-\pi)$. If $\gamma$ does not already self-intersect between $-\pi$ and $\pi$, then one of the two branches is under the other one (see figure 2). Say $\operatorname{Im}[\gamma(\pi)] \leq \operatorname{Im}[\gamma(-\pi)]$. In that case, a sufficient condition for $\gamma([\pi, 3 \pi / 2])$ to intersect $\gamma([-\pi, 0])$ is $\operatorname{Re}[\gamma(-\pi / 2)] \geq \operatorname{Re}[\gamma(3 \pi / 2)]$ by continuity. Namely, from $\pi$ to $3 \pi / 2, \operatorname{Re}[\gamma]$ decreases at least from:

$$
\int_{\pi}^{3 \pi / 2} \frac{\cos \theta}{\kappa(\theta)} \leq \int_{\pi}^{3 \pi / 2} \frac{\cos \theta}{\kappa(\pi)}=-\frac{1}{\kappa(\pi)}<0
$$

Conversely, $\operatorname{Re}[\gamma(-\pi / 2)]$ is bounded below by $\frac{-1}{\kappa(-\pi / 2)}$. Hence the condition (iii).

\section{An analytical criterion for self-intersection}

We now give an analytical criterion on the holomorphic Gauss map $g$ for self-intersection, using the previous geometrical criterion.

Proposition 4 If there exists a sequence of $z_{n}$ going to infinity such that

(i) $\left|g\left(z_{n}\right)\right|=1$,

(ii) Let $e^{i \theta_{n}}=h\left(z_{n}\right) /\left|h\left(z_{n}\right)\right|$; then $e^{i \theta_{n}}$ is different from $\pm 1, \pm i$,

(iii) $\max _{|t| \leq \frac{2 \pi}{\sin \theta_{n}\left|h\left(z_{n}\right)\right|}, t \in \mathbb{R}}\left|\frac{h^{\prime}\left(z_{n}+t\right)}{h^{2}\left(z_{n}\right)}\right| \in o\left(\sin ^{2} \theta_{n} \cos ^{2} \theta_{n}\right)$

then lemma 3 applies.

PROOF: The key idea is to make an appropriate change of variable (or "zooming") around the $z_{n}$. We will prove that close to $z_{n}$, there is a maximum or minimum of the curvature, while the tangent angle varies enough around $z_{n}$ to satisfy the conditions of lemma 3.

Close to $z_{n}, g$ does not vanish, hence we can integrate $h$ into $H$, and write $g=e^{H}$ with the assumption that $\operatorname{Re}\left[H\left(z_{n}\right)\right]=0$. We now make the following change of variable: $u=\sin \theta_{n}\left|h\left(z_{n}\right)\right| t$, and denote $\hat{H}(u)=H\left(z_{n}+t(u)\right), \hat{h}(u)=d \hat{H} / d u=\frac{h\left(z_{n}+t(u)\right)}{\sin \theta_{n}\left|h\left(z_{n}\right)\right|}$. Asymptotically, $u$ is the tangent angle. We rewrite the hypotheses at $z_{n}$ as:

(i') $\operatorname{Re}[\hat{H}(0)]=0$

(ii') $\quad e^{i \theta_{n}}=\sin \theta_{n} \hat{h}(0)$ and $e^{i \theta_{n}}$ is different from $\pm 1, \pm i$.

(iii') $\max _{u \in[-2 \pi, 2 \pi]}\left|\hat{h}^{\prime}(u)\right| \in o\left(\cos ^{2} \theta_{n}\right)$ 
By integrating (iii') one derives the following inequalities:

$$
\begin{aligned}
& \max _{u \in[-2 \pi, 2 \pi]}\left|\int_{0}^{u} \hat{h}^{\prime}(v) d v\right| \in o\left(\cos ^{2} \theta_{n}\right) \\
& \max _{u \in[-2 \pi, 2 \pi]}\left|\int_{0}^{u} \int_{0}^{v} \hat{h}^{\prime}(w) d w d v\right| \in o\left(\cos ^{2} \theta_{n}\right)
\end{aligned}
$$

We now write the expression for the curvature and its derivative:

$$
\begin{aligned}
& \frac{1}{2} \kappa(u)=\frac{\operatorname{Im}[\hat{h}]}{\cosh \operatorname{Re}[\hat{H}]} \\
& \frac{1}{2} \frac{d \kappa}{d u}=\frac{\operatorname{Im}\left[\hat{h}^{\prime}(u)\right]-\operatorname{Re}[\hat{h}] \operatorname{Im}[\hat{h}] \tanh (\operatorname{Re}[\hat{H}])}{\cosh \operatorname{Re}[\hat{H}]}
\end{aligned}
$$

Let us write the expansion of the above quantities, taking $u$ to be bounded (say $|u| \leq 2 \pi$ ):

$$
\begin{aligned}
& \hat{H}(u)=H\left(z_{n}\right)+\left(\cot \theta_{n}+i\right) u+R(u) \\
& R(u)=\int_{0}^{u} \int_{0}^{v} \hat{h}^{\prime}(w) d w d v \\
& \operatorname{Re}[\hat{H}(u)]=\cot \theta_{n} u+\operatorname{Re}[R(u)] \\
& \tanh (\operatorname{Re}[\hat{H}(u)])=\cot \theta_{n} u+\operatorname{Re}[R(u)]+o\left(u^{2}+|R|^{2}(u)\right)
\end{aligned}
$$

then the numerator of $\frac{1}{2} d \kappa / d u$ is:

$$
\begin{aligned}
& \operatorname{Im}\left[R^{\prime \prime}(u)\right]-\left(\cot \theta_{n}+\operatorname{Re}\left[R^{\prime}(u)\right]\right)\left(1+\operatorname{Im}\left[R^{\prime}(u)\right]\right)\left(\cot \theta_{n} u+\operatorname{Re}[R(u)]+o\left(u^{2}+|R|^{2}(u)\right)\right. \\
& =\operatorname{Im}\left[R^{\prime \prime}(u)\right]-\cot ^{2} \theta_{n} u+o\left(\cot ^{2} \theta_{n}\right)
\end{aligned}
$$

because $u$ is bounded. That implies that $d \kappa / d u$ vanishes at some point $u_{0}$ within the bounds, for $n$ big enough. Indeed hypothesis (ii') guarantees that $\cot \theta_{n}$ is well-defined and different from zero; thus

$$
\left|u_{0}-\frac{R^{\prime \prime}\left(u_{0}\right)}{\cot ^{2} \theta_{n}}\right| \leq o(1)
$$

Since actually $R^{\prime \prime}(u) \tan ^{2} \theta_{n} \in o\left(\sin ^{2} \theta_{n}\right), u_{0} \underset{n \rightarrow \infty}{\longrightarrow} 0$.

Now that we have a local maximum at $u_{0}$ for $\kappa(u)$, there remains to prove the growth conditions for lemma 3 . Let $u_{k}$ be the first time that the tangent angle $\operatorname{Im}[\hat{H}]$ hits the value $\operatorname{Im}\left[\hat{H}\left(u_{0}\right)\right]+k \pi / 2$ for some integer $k$ :

$$
\frac{k \pi}{2}=\left(u_{k}-u_{0}\right)+\operatorname{Im}\left[R\left(u_{k}\right)-R\left(u_{0}\right)\right]
$$

Obviously for $|k| \leq 3$ and large $n, u_{k}$ remains within the bounds of the hypotheses, and we are entitled to write

$$
u_{k}=\frac{k \pi}{2}+o\left(\cos ^{2} \theta_{n}\right) \sim \frac{k \pi}{2}
$$


Clearly $\kappa$ increases on $\left[u_{-3}, u_{0}\right]$ and decreases on $\left[u_{0}, u_{3}\right]$. We finally evaluate

$$
\kappa\left(u_{k}\right)=\frac{1+\operatorname{Im}\left[R^{\prime}(u)\right]}{\cosh \left(\cot \theta_{n}\left(u_{k}+o(1)\right)\right)}=\frac{1+\operatorname{Im}\left[R^{\prime}(u)\right]}{\cosh \left(\cot \theta_{n}(k \pi / 2+o(1))\right)}
$$

Since cosh is an even function that increases on $\mathbb{R}_{+}$, we get

$$
\frac{\cosh \left(\cot \theta_{n}(\pi+o(1))\right)}{\cosh \left(\cot \theta_{n}(\pi / 2+o(1))\right)}>1
$$

for large $n$. So $\kappa\left(u_{-2}\right)<\kappa\left(u_{1}\right)$ and $\kappa\left(u_{2}\right)<\kappa\left(u_{-1}\right)$.

The assertion (iii) of the proposition 4 can be readily modified into a more usable condition.

Lemma 5 Let $\phi$ be any meromorphic function on the plane, $\left(z_{n}\right)$ a given sequence of complex numbers, and $\left(\epsilon_{n}\right),\left(K_{n}\right)$ two sequences of non-negative real numbers, such that $K_{n} \epsilon_{n} \underset{n \rightarrow \infty}{\longrightarrow} 0$. Then (a) implies (b):

$$
\begin{aligned}
& \text { (a) } \max _{\left|z-z_{n}\right| \leq \frac{K_{n}}{\left|\phi\left(z_{n}\right)\right|}}\left|\frac{\phi^{\prime}(z)}{\phi(z)^{2}}\right| \in O\left(\epsilon_{n}\right) \\
& \text { (b) } \max _{\left|z-z_{n}\right| \leq \frac{K_{n}}{\left|\phi\left(z_{n}\right)\right|}}\left|\frac{\phi^{\prime}(z)}{\phi\left(z_{n}\right)^{2}}\right| \in O\left(\epsilon_{n}\right)
\end{aligned}
$$

PROOF: Since

$$
\frac{\phi^{\prime}(z)}{\phi\left(z_{n}\right)^{2}}=\frac{\phi^{\prime}(z)}{\phi(z)^{2}}\left(\frac{\phi(z)}{\phi\left(z_{n}\right)}\right)^{2}
$$

there remains to estimate $\left|\phi(z) / \phi\left(z_{n}\right)\right|$ for $\left|z-z_{n}\right| \leq K_{n} /\left|\phi\left(z_{n}\right)\right|$. If (a) is true, the derivative $\frac{d}{d z}(1 / \phi)$ is bounded by $O\left(\epsilon_{n}\right)$ on that same interval; thus:

$$
\left|\frac{1}{\phi(z)}-\frac{1}{\phi\left(z_{n}\right)}\right| \leq\left|z-z_{n}\right| O\left(\epsilon_{n}\right) \leq \frac{K_{n}}{\left|\phi\left(z_{n}\right)\right|} O\left(\epsilon_{n}\right)
$$

so

$$
\left|\frac{\phi\left(z_{n}\right)}{\phi(z)}-1\right| \leq O\left(K_{n} \epsilon_{n}\right) \underset{n \rightarrow \infty}{\longrightarrow} 0
$$

We apply lemma 5 to proposition 4 with $\phi=h=g^{\prime} / g, K_{n}=2 \pi / \sin \theta_{n}$ and $\epsilon_{n}=\delta_{n} \sin ^{2}\left(2 \theta_{n}\right)$ with $\delta_{n} \underset{n \rightarrow \infty}{\longrightarrow} 0$. The condition $K_{n} \epsilon_{n} \underset{n \rightarrow \infty}{\longrightarrow} 0$ is obviously satisfied. Hence we can replace condition (iii) with an analytically more satisfying criterion. 


\section{Functions of finite order of growth}

We now turn to functions $g$, meromorphic in a neighborhood of infinity $D_{r_{0}}^{\infty}=\{z \in \mathbb{C} ;|z| \geq$ $\left.r_{0}\right\}$. We define as usual (see [3] for instance) $A(r)$ to be the area of the image of $r_{0} \leq|z| \leq r$ by $g$ on the Riemann sphere, counted with multiplicity, divided by $4 \pi$. Then the characteristic function (according to Ahlfors-Shimizu) for $g$ is:

$$
T(r)=\int_{r_{0}}^{r} \frac{A(t) d t}{t}
$$

and the growth order of $g$ is equal to:

$$
\lambda=\varlimsup_{r \rightarrow \infty} \frac{\log T(r)}{\log r}
$$

We use this terminology and the following

Lemma 6 If $g$ is a non-vanishing holomorphic function on $D_{r_{0}}^{\infty}$ and $g$ has finite order of growth in the sense defined above, then $h=g^{\prime} / g$ extends meromorphically at infinity (in particular $|h(z)| \in O\left(z^{k}\right)$ for some positive $\left.k\right)$.

PROOF: for a beautiful self-contained proof of this, see [11].

Finally we derive the main result:

PROOF OF THE THEOREM : Using the previous lemma, we conclude that $h$ can be written with a Laurent expansion around infinity containing a finite number of positive exponents:

$$
h(z)=\sum_{k=-\infty}^{k=p} a_{k} z^{k}
$$

We proceed to prove the following:

- Hypothesis (iii) of the analytic criterion (proposition 4) is always satisfied, provided $p \geq 0$ (if not see below). Indeed, using lemma 5 we simply consider the derivative of $1 / h$, which vanishes at infinity. Thus the hypothesis is strongly satisfied, indeed

$$
\max _{t \in \mathbb{R}}\left|\frac{h^{\prime}\left(z_{n}+t\right)}{h\left(z_{n}+t\right)^{2}}\right| \in O\left(z^{-p-1}\right)
$$

for any sequence $z_{n}$ such that $\left|\operatorname{Im} z_{n}\right| \underset{n \rightarrow \infty}{\longrightarrow} \infty$.

- There remains to find such a sequence with appropriate angle conditions. Suppose $p \geq 1$. We write $z_{n}$ as a zero of $\log |g|=\operatorname{Re} \int^{z}[h]$ :

$$
\log |g|=\sum_{k \leq p, k \neq-1} \operatorname{Re}\left[\frac{a_{k}}{k+1} z^{k+1}+a_{-1} \log z\right]=\operatorname{Re}\left[\frac{a_{p}}{p+1} z^{p+1}\right]\left(1+O\left(\frac{1}{z}\right)\right)
$$


It will soon be obvious that the exact modulus of $a_{p}$ does not matter, so let us assume that $a_{p}=e^{i \beta}\left|a_{p}\right|$. Then the argument $\alpha_{n}$ of $z_{n}$ may take the following values:

$$
\alpha_{n} \equiv \frac{\pi}{2(p+1)}-\frac{\beta}{p+1}+o(1) \bmod \frac{\pi}{p+1}
$$

Consider now $e^{i \theta_{n}}=h\left(z_{n}\right) /\left|h\left(z_{n}\right)\right|$. Since $h(z) \sim a_{p} z^{p}$, so $\theta_{n}$ is congruent to $\beta+p \alpha_{n}+$ $o(1) \bmod 2 \pi$ :

$$
\theta_{n} \equiv \frac{\beta}{p+1}+\frac{p \pi}{2(p+1)}+o(1) \bmod \frac{p \pi}{p+1}
$$

The adherence of the $\theta_{n}$ is contained in $\frac{\beta}{p+1}+\frac{p \pi}{2(p+1)}+\frac{p \pi}{p+1} \mathbb{Z}$, which cannot be included into any $\frac{\pi}{2} \mathbb{Z}$, unless $\frac{4 p \pi}{p+1} \equiv 0 \bmod 2 \pi$. That may happen only if $p=1$, and $\beta=\pi / 2 \bmod$ $\pi$. That case has a very particular behavior and requires a special proof; see lemma 7 below. Otherwise we deduce easily that the $e^{i \theta_{n}}$ stay uniformly away from $\pm 1, \pm i$, for some sequence $z_{n}$ satisfying $\left|g\left(z_{n}\right)\right|=1$. We conclude using proposition 4 . See figure 3 for an example of the loops, existing for most level curves.

- If $p=0$, we have something that is very much like the helicoid or one of its conjugates: $g(z)=z^{k} e^{a_{0} z+c+O(1 / z)}$ for some integration constant $c$. Thus

$$
B(t)=\arg [g(t+i l)]=\operatorname{Im}\left[a_{0}\right] t+\operatorname{Re}\left[a_{0}\right] l+k \arg (t+i l)+\operatorname{Im}[c]+O(1 / z)
$$

If $\operatorname{Im}\left[a_{0}\right] \neq 0, \operatorname{deg}(B)$ is odd, and using remark 1 (page 5 ) we see that the surface selfintersects obviously. If on the contrary $a_{0}$ is real, a necessary condition for embeddedness is $|k| \leq 1$ (otherwise, for large $l, B(t)$ would be increasing and span $k \pi$, thus forcing self-intersection). If $k= \pm 1$, this argument cannot be applied, and the upper (or lower) half-plane is embedded; but not the whole surface, which is not well defined, because there is a period around the end ${ }^{3}$. Indeed we show that

$$
\int_{|z|=r}\left(g+g^{-1}\right) d z=2 \pi i e^{ \pm c}
$$

Say $k=-1$ (otherwise take $g^{-1}$ ), then

$$
\int_{|z|=r} g d z=e^{c} \int_{|z|=r} e^{a_{0} z}(1+O(1 / z)) \frac{d z}{z} \sim e^{c} \int_{|z|=r} e^{a_{0} z} \frac{d z}{z} \quad \text { as } r \rightarrow \infty
$$

Since $e^{a_{0} z} / z$ extends to the whole complex plane, we can evaluate its residue at zero, and conclude. There remains only the possibility of $k=0$, as in the case of the helicoid. Such an end can exist and be embedded, two possibilities exist: either the unknown term $O(1 / z)$ vanishes completely, and we have exactly a helicoid, or there is a non-zero term in the Laurent expansion, and up to simple reparametrization, $g(z)=e^{z} e^{\phi(z)}$ where

\footnotetext{
${ }^{3}$ It is obvious from the Weierstrass-Enneper representation that a residue for $g$ or $g^{-1}$ at any point means a period for $X_{1}$ or $X_{2}$ around that point.
} 


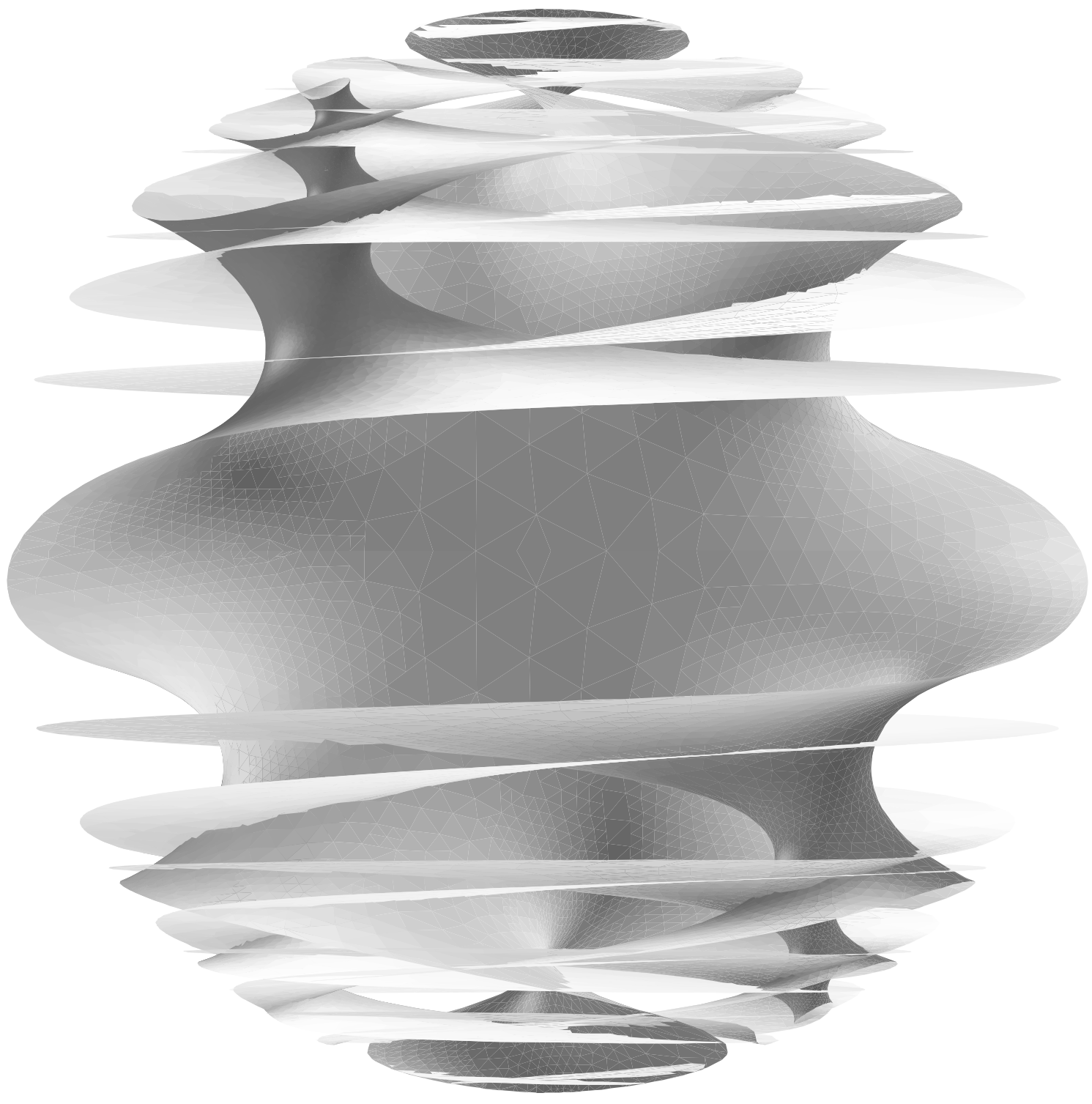

Figure 3: example of application of the geometric criterion: surface with imbricated spirals for $g=e^{z^{3}}$, where almost all level curves make a self-intersection loop. 
$\phi(z)=a z^{-n}+O\left(z^{-n-1}\right)$. Such surfaces exist and are embedded; their level curves have an asymptotic direction similar to the helicoids, but they are not asymptotic to lines. Indeed

$$
\dot{\xi}_{l}=\frac{d \xi_{l}}{d t}=-\frac{i}{2} \cosh (t+\operatorname{Re}[\phi]) e^{i(l+\operatorname{Im}[\phi])}
$$

and

$$
\frac{\dot{\xi}_{l}}{\left|\dot{\xi}_{l}\right|} \underset{t \rightarrow \pm \infty}{\longrightarrow}-i e^{i l}
$$

while

$$
\left\langle\frac{d \xi_{l}}{d t}, e^{i l}\right\rangle=\frac{1}{2} \cosh (t+\operatorname{Re}[\phi]) \sin \operatorname{Im}[\phi]
$$

notice now that $\operatorname{Im}[\phi]$ is asymptotically greater than $t^{-n-1}$ times a constant, and $\int_{0}^{\infty} \cosh t \sin t^{-n-1} d t$ is a diverging integral. So theses examples are qualitatively different from the helicoid. They are embedded at the end though because of the asymptotic behaviour just quoted above.

- If $p$ is actually less than zero, then the surface has finite total curvature, and is necessarily the plane. Notice indeed that the end is not horizontal (since $X_{3} \rightarrow \pm \infty$ when $|z| \rightarrow \infty$ ). So the Gauss map has a finite non-zero limit at infinity, in particular $k=0$. It is branched, since $\lim _{|z| \rightarrow \infty} h(z)=0$ by hypothesis. The end cannot be catenoidal, is it asymptotic to a plane.

Lemma 7 With all the previous notations, suppose that $h(z) \sim a_{1} z$ and $a_{1}$ is pure imaginary; then the surface is not embedded and actually not even proper.

PROOF : We write $g=e^{H}$ where $H(z)=a_{1} z^{2} / 2+a_{0} z+k \log z+c+o(1)$, where $c$ is an integration constant. Up to translation and dilation in $\mathbb{C}$, we can assume that $a_{1}=i$ and $a_{0}=0$. We will construct a path on the surface which goes to infinity in $\mathbb{C}$ but stays at bounded distance in $\mathbb{R}^{3}$. Let $\gamma(s)$ denote a path in $\mathbb{C}$, not necessarily horizontal this time. Still writing $A=\operatorname{Re}[H]$ and $B=\operatorname{Im}[H]$, we have the following equation for $\xi=X_{1} \circ \gamma+i X_{2} \circ \gamma$ :

$$
i \frac{d \xi}{d s}=e^{i B}\left(e^{A} \gamma^{\prime}+e^{-A} \overline{\gamma^{\prime}}\right)
$$

We would like $A \circ \gamma$ to be bounded, knowing that $A\left(r e^{i \theta}\right)=-r^{2} \sin 2 \theta+k \log r+c+o(1)$ ( $c$ denotes any constant). If we choose $\theta$ such that $\sin 2 \theta=\frac{k \log r}{r^{2}}$ as $r$ goes to infinity, and denote by $\gamma(s)$ that curve parameterized by its arclength (for the standard metric in $\mathbb{C}$ ); then $A(\gamma(s)) \sim c+o(1)$. Notice also that $l(s)=\operatorname{Im}[\gamma(s)] \underset{t \rightarrow \infty}{\longrightarrow} 0, t(s)=\operatorname{Re}[\gamma(s)]=s+o(1)$ and $\gamma^{\prime}(s) \underset{s \rightarrow \infty}{\longrightarrow} 1$. Thus

$$
i \frac{d \xi}{d s}=C_{1} e^{i t(s)^{2} / 2}(\cosh c+o(1))=C_{2} e^{i s^{2} / 2}+o(1)
$$

$C_{1}, C_{2}$ appropriate complex constants. But this integral is definite, indeed

$$
\int_{0}^{S} e^{i s^{2} / 2} d s=\int_{0}^{S^{2}} \frac{e^{i u}}{\sqrt{2 u}} d u
$$

The surface is not properly immersed (see figure 4). 


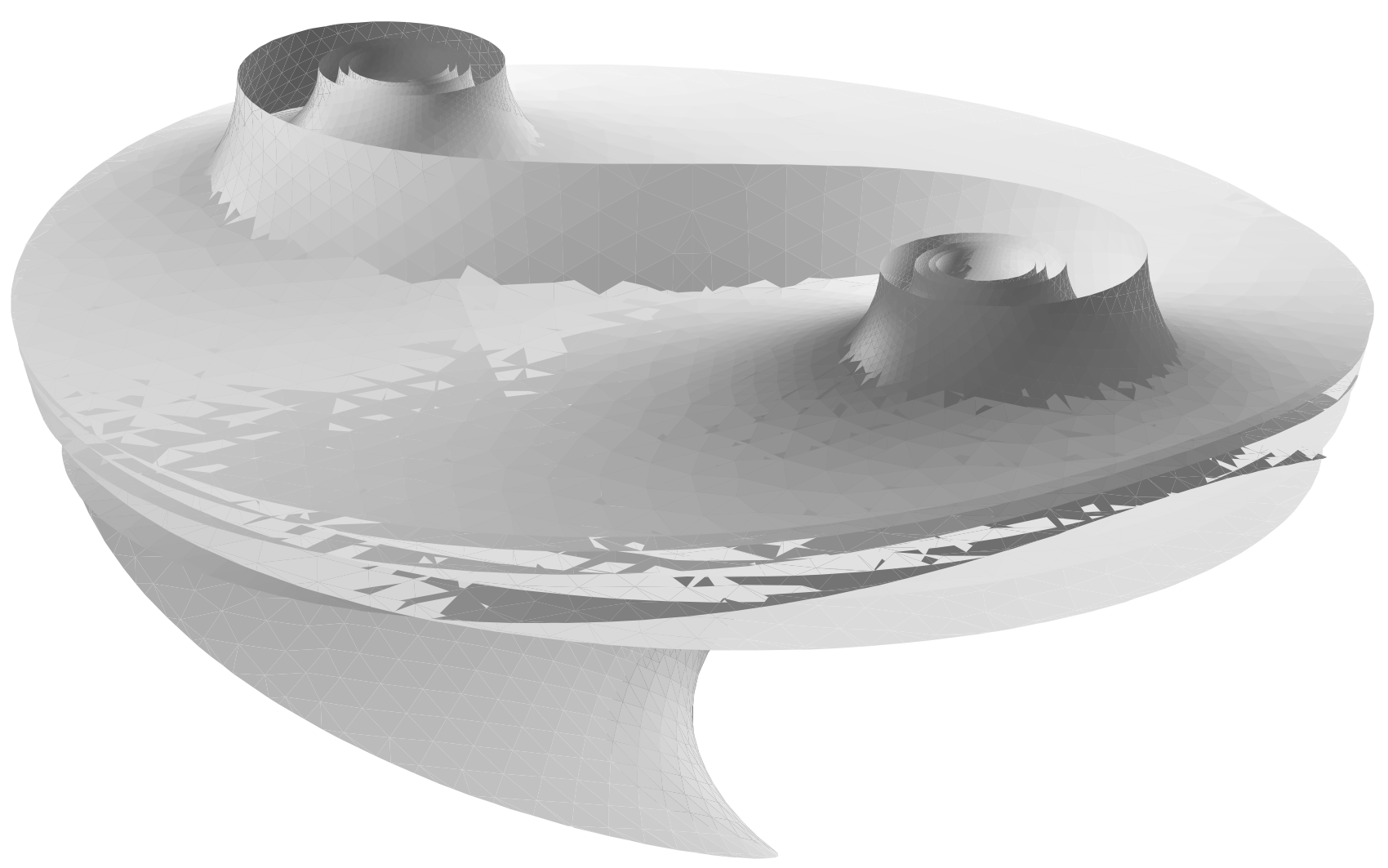

Figure 4: An example of non proper minimal immersion for $g=e^{i z^{2}}$ (only the points with negative third coordinate are shown). The level curve for $X_{3}=0$ does not go to infinity. 
PROOF OF THE COROLLARY : We have a one-ended minimal surface, conformally equivalent to $\mathbb{C}$. Since is has only one end, the surface cannot have finite total curvature. The general form of the Gauss map is

$$
g(z)=R(z) e^{\phi(z)}
$$

for some entire function $\phi$ and rational function $R$ having poles and zeros where $X_{3}$ has a critical point (horizontal tangent plane). For some integer $k, z^{-k} R(z) \underset{z \rightarrow \infty}{\longrightarrow} C \neq 0, \infty$, so we can rewrite $R(z)$ as $C z^{k} e^{\psi(z)}$ with $\psi$ holomorphic on $D_{r}^{\infty}$ for large $r$. Applying the theorem, we know that $k=0$ and the Gauss map can be written as

$$
g(z)=C e^{z} e^{\chi(z)}
$$

where $\chi(z) \in O(1 / z)$ is entire. So $\chi$ is identically zero, we have a helicoid.

\section{Acknowledgments}

I am grateful to Harold Rosenberg for teaching me all the beauty of minimal surfaces, and particularly for his suggestion of the proof of lemma 1. I also thank David Hoffman and the people at the Center for Geometry, Analysis, Numerics and Graphics, especially Jim Hoffman, David Oliver and Ed Thayer for helping me to create such striking and inspiring pictures. Finally I thank my colleagues at the CMLA (Centre de Mathématiques et Leurs Applications) for their support in the making and writing of this article. 


\section{References}

[1] P. Collin : Topologie et courbure des surfaces minimales de $\mathbb{R}^{3}$, personal communication.

[2] G. CRow : On a conjecture of Nitsche, AMS Proceedings 114-4 (1992), 1063-1068.

[3] W. K. Hayman : Meromorphic functions, Oxford Mathematical Monographs.

[4] D. Hoffman, H. Karcher and F. Wei : Adding handles to the helicoid, AMS Bulletin, New Series 29-1 (1993), 77-84.

[5] H. B. LaWson : Lectures on minimal submanifolds, Vol. 1. Math. Lecture Series 9, Publish or Perish.

[6] W. H. Meeks III, H. Rosenberg : The geometry of periodic minimal surfaces, Comment Math. Helv. 68-4 (1993), 538-578.

[7] R. Osserman : A survey of minimal surfaces, Van Nostrand Reinhold Math., 25, 1969.

[8] P. Romon : Quelques aspects de la théorie des surfaces minimales dans $\mathbb{R}^{3}$, Thesis, Université Paris VII, 1993.

[9] P. Romon : A rigidity theorem for Riemann's minimal surfaces, Annales de l'Institut Fourier 43 (1993), 485-502.

[10] H. Rosenberg : Some recent developments in the theory of properly embedded minimal surfaces in $\mathbb{R}^{3}$. Séminaire Bourbaki $\mathbf{7 5 9}$ (1992).

[11] H. Rosenberg : Minimal surfaces of finite type (to appear).

[12] H. Rosenberg, E. Toubiana : A cylindrical type complete minimal surface in a slab of $\mathbb{R}^{3}$, Bulletin Sc. Math 111 (1987), 241-245.

[13] W. Rudin : Real and complex analysis, Mac Graw-Hill. 